EPJ Web of Conferences 35, 06004 (2012)

DOI: $10.1051 /$ epjconf/20123506004

(c) Owned by the authors, published by EDP Sciences, 2012

\title{
Decay of a three-quasiparticle isomer in the neutron-rich nucleus ${ }^{183} \mathrm{Ta}$
}

\author{
N. Palalani ${ }^{1, a}$, G.J. Lane ${ }^{1}$, G.D. Dracoulis ${ }^{1}$, F.G. Kondev ${ }^{2}$, A.P. Byrne ${ }^{1}$, M.P Carpenter ${ }^{2}$, C.J. Chiara ${ }^{2}$, P. Chowdhury ${ }^{3}$, \\ R.O. Hughes ${ }^{1}$, R.V.F. Janssens ${ }^{2}$, T. Lauritsen ${ }^{2}$, C.J. Lister ${ }^{2}$, E.A. McCutchan ${ }^{2}$, D. Seweryniak ${ }^{2}$, I. Stefanescu², \\ H. Watanabe ${ }^{1}$, and S. Zhu ${ }^{2}$ \\ 1 Department of Nuclear Physics, The Australian National University, ACT 0200, Australia. \\ 2 Argonne National Laboratory, Argonne, Illinois 60439, USA \\ 3 University of Massachusetts Lowell, Lowell, Massachusetts 01854, USA
}

\begin{abstract}
Excited states in neutron-rich tantalum isotopes have been studied with deep-inelastic reactions using ${ }^{136} \mathrm{Xe}$ ions incident on a ${ }^{186} \mathrm{~W}$ target. New transitions observed below the $\tau=1.3 \mu$ s isomer in ${ }^{183} \mathrm{Ta}$ have enabled the establishment of its energy and put limits on the spin and parity. On the basis of the reduced hindrances for the depopulating transitions, a 3-quasiparticle configuration of $v 1 / 2^{-}[510] 11 / 2^{+}[615] \otimes \pi 9 / 2^{-}[514]$ is suggested.
\end{abstract}

\section{Introduction}

Nuclei in regions far from closed shells are known to have non-spherical equilibrium shapes which are either oblate, or, more usually, prolate. The single particle energy levels in these nuclei depend on the component of the nucleon angular momentum (denoted by $j_{z}=\Omega$ ) along the symmetry axis. These components can be summed over the valence nucleons to give a quantum number $K=\sum_{i} \Omega_{i}$ that is nominally conserved. Nuclei around mass-180 exhibit metastable states known as K-isomers [1,2], that form when states with high $\mathrm{K}$ values can only decay via transitions whose multipole order $\lambda$ is less than the change in $\mathrm{K}$, thus violating the $\mathrm{K}$-selection rule, $\Delta K \leq \lambda$. Such transitions are, in principle, forbidden, with the forbiddenness characterised by $v=\Delta \mathrm{K}-\lambda$.

Experimental access to $\mathrm{K}$-isomers that are expected to occur in the neutron-rich region has been limited by the lack of stable beams and targets that can populate them using conventional fusion-evaporation reactions. Recently, $\mathrm{K}$ isomers in the neutron-rich hafnium, lutetium and tungsten isotopes [3-8] have been studied using more exotic techniques such as deep-inelastic reactions [9] and relativistic fragmentation [10]. The present report focusses on the tantalum isotopes and, in particular, on ${ }^{183} \mathrm{Ta}$.

The heaviest stable tantalum nucleus is ${ }^{181} \mathrm{Ta}$ and little is known concerning high spin states in isotopes heavier than ${ }^{183} \mathrm{Ta}$, except for the decay of the $\mathrm{K}^{\pi}=21 / 2^{-}$isomer in ${ }^{185} \mathrm{Ta}[11,12]$. Recently, Shizuma et al [13] investigated ${ }^{183} \mathrm{Ta}$ using the ${ }^{181} \mathrm{Ta}\left({ }^{18} \mathrm{O}{ }^{16} \mathrm{O}\right){ }^{183} \mathrm{Ta}$ transfer reaction and reported the decay from a new isomeric state with a $1.3 \mu$ s lifetime. The tentative spin, parity and configuration assignments were based on the assumption that an unobserved transition depopulated the isomer. At present no information on high spin states is available for ${ }^{184} \mathrm{Ta}$. The current work aims to extend the level schemes for these neutron-rich tantalum nuclei to high-spin using deepinelastic reactions. This forms part of an ongoing pro-

\footnotetext{
a e-mail: nyaladzi.p@gmail.com
}

gram to explore the structures of well deformed neutronrich nuclei (see, for example, $[4,6,7,14,15]$ and references therein). In the current work, additional transitions below the $1.3 \mu$ s isomer in ${ }^{183}$ Ta have been identified, so that the energy and likely multipolarity of the unobserved transition, and hence the energy and the configuration for the isomer, have been obtained. While states above the isomer have also been observed, including two new isomers, and $\gamma$-rays possibly associated with ${ }^{184} \mathrm{Ta}$ have been identified, the analysis of these is still in progress and will not be discussed in the present report.

\section{Experimental Methods}

The current results are from data collected at Argonne National Laboratory using Gammasphere with 99 Comptonsuppressed HPGe detectors in operation. A beam of $840 \mathrm{MeV}{ }^{136} \mathrm{Xe}$ ions from the ATLAS accelerator was incident on a $99 \%$ enriched ${ }^{186} \mathrm{~W}$ target with a gold backing. This reaction is expected to populate tantalum nuclei from $\mathrm{A} \approx 182$ to $\mathrm{A} \approx 187$. The experimental measurements involved a variety of macroscopic beam pulsing conditions, ranging from microseconds to seconds, to enable identification of isomers with a range of lifetimes. The data were gain-matched offline for each individual detector and sorted into a Blue database [16] for time correlated coincidence analysis.

\section{Experimental Results}

\section{$3.1^{183}$ Ta level scheme}

Several gate combinations were set in the out-of-beam time region on $\gamma$-rays below the isomer in ${ }^{183} \mathrm{Ta}$. All the known $\gamma$-rays from Ref. [13] were observed, together with new $\mathrm{J} \rightarrow \mathrm{J}$ transitions at 488,473 and $442 \mathrm{keV}$ that decay from the $13 / 2^{-}$band to the $9 / 2^{-}$band. The new transitions extend 


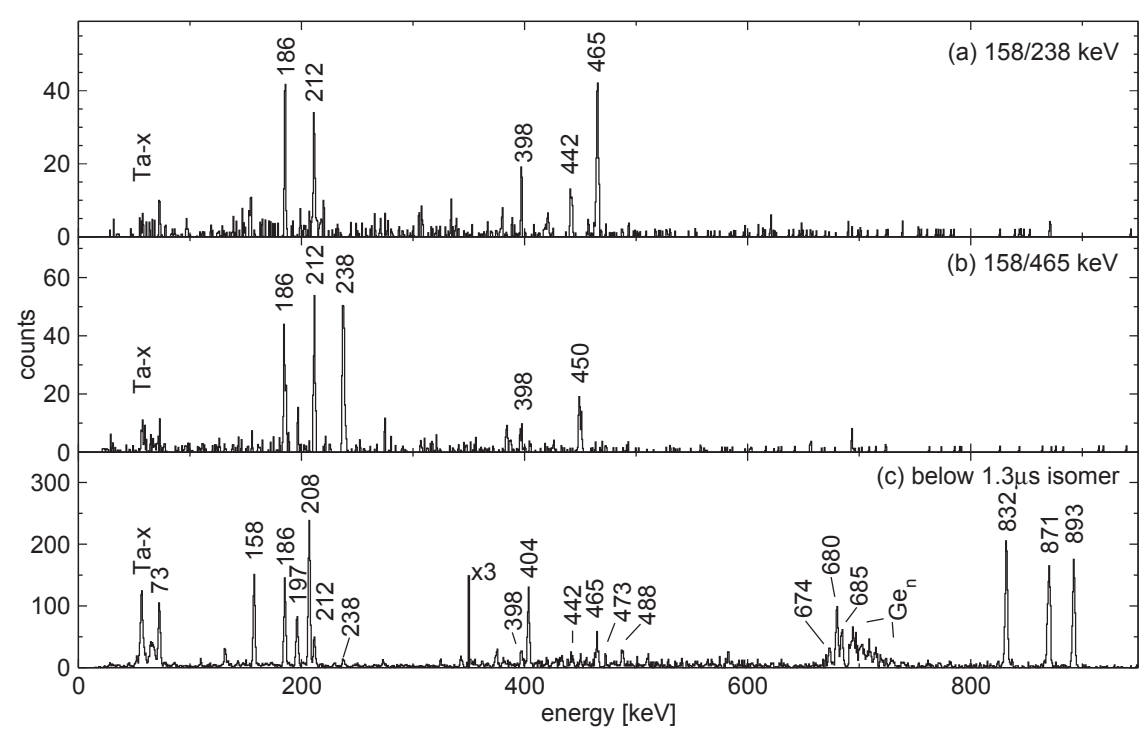

Fig. 1. (a) and (b) Coincidence spectra projected from the out-of-beam $\gamma-\gamma-\gamma$ coincidence cube double gated on 158/238 and $158 / 465 \mathrm{keV}$ transitions, respectively. Spectrum (a) shows the new 442 and $465 \mathrm{keV}$ transitions, while spectrum (b) demonstrates that they arise from parallel decay paths. (c) Delayed $\gamma$-ray spectrum showing the transitions that follow the decay of the $1.3 \mu$ s isomer (see text for details). Contaminants due to inelastic neutron excitation in ${ }^{72} \mathrm{Ge}$ are labelled.

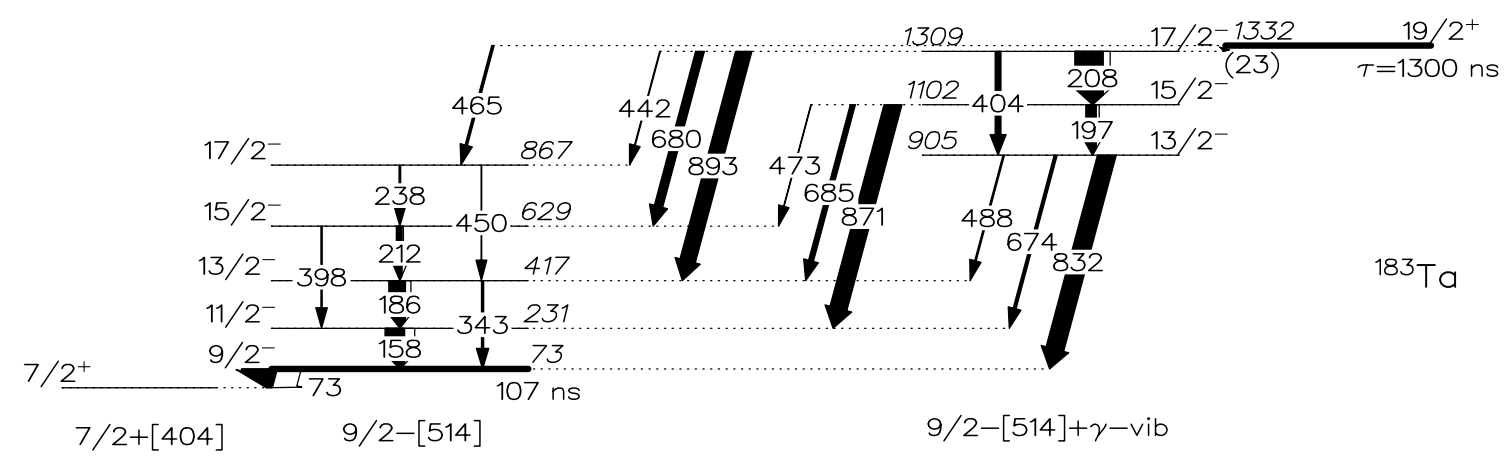

Fig. 2. Level scheme for ${ }^{183} \mathrm{Ta}$ including new transitions that establish the energy of the unobserved $23 \mathrm{keV}$ transition.

the decay pattern expected for the $13 / 2^{-}$band if it were to be the $9 / 2^{-}$configuration coupled to the $\gamma$-vibration, as already suggested by Shizuma et al [13]. In addition, a $465 \mathrm{keV}$ direct decay from the isomer was identified. A representative double-gated spectrum comprising gates on known transitions in the $9 / 2^{-}[514]$ band $(158$ and $238 \mathrm{keV})$ is shown in Figure 1.

The level scheme for ${ }^{183} \mathrm{Ta}$ below the $1.3 \mu \mathrm{s}$ isomer as deduced from the coincidence information is illustrated in Figure 2. The structure below the isomeric state reported by Shizuma et al [13] is confirmed, and new transitions included. The spectrum in Figure 1(b) demonstrates that two of the new transitions are parallel and implies that the $465 \mathrm{keV}$ transition directly depopulates the isomer. From this information, the energy of the unobserved transition previously proposed in Ref. [13] is deduced to be $23 \mathrm{keV}$.

By double-gating on transitions below the isomer and projecting the $\gamma$-rays that precede it in time, a new set of feeding $\gamma$-rays was identified. While these $\gamma$-rays are not presented here, they were used to isolate the delayed $\gamma$ rays emitted below the $1332 \mathrm{keV}$ state. The 200 to $800 \mathrm{~ns}$ delayed spectrum in Figure 1(c) was used to deduce the $\gamma$-ray intensities for decay branches out of the isomer.

\section{Discussion}

The ground state band is known to be formed from the $7 / 2^{+}[404]$ orbital, while the first excited band is built on the $9 / 2^{-}[514]$ intrinsic state. The $13 / 2^{-}$bandhead is suggested in Ref [13] to be a $\gamma$-vibration coupled to the $9 / 2^{-}[514]$ state. Therefore, the following discussion is focused on the establishment of the spin, parity and single-particle configuration for the $1332 \mathrm{keV}$ isomer.

\subsection{Spin assignment for the $1332 \mathrm{keV}$ state}

To determine the multipolarity of the $23 \mathrm{keV}$ transition, several possible spins and parities for the $1332 \mathrm{keV}$ state were assumed. For each case, the implied multipolarities of the 23 and $465 \mathrm{keV}$ transitions were deduced, and $\gamma$-ray intensities were determined based on measured $\gamma$-ray intensities, intensity balances and internal conversion coefficients. The transition strengths and reduced hindrances for the 465 and $23 \mathrm{keV}$ transitions thus obtained are shown in Table 1. Since E1 transitions are already hindered by large factors, the reduced hindrances for E1 transitions were calculated both with (italics) and without an additional factor of $10^{4}$ in the nominal single particle hindrance. In the absence of competing mechanisms for enhanced decays, ex- 
Table 1. Transition strengths and corresponding reduced hindrances for the 465 and $23 \mathrm{keV}$ transitions, assuming alternative spins and parities for the $1.3 \mu$ s isomeric state.

\begin{tabular}{cccccccc}
\hline \hline$I^{\pi}$ & $\lambda$ & $E_{\gamma}(\mathrm{keV})$ & $I_{\gamma}$ & $\alpha_{T}$ & $B_{\gamma}(\mathrm{W} . \mathrm{u})$ & $v$ & $f_{v}$ \\
\hline $19 / 2^{-}$ & $M 1$ & $(22.7)^{a}$ & $(74.3)^{b}$ & $5.771 \times 10^{1}$ & $3.46 \times 10^{-5}$ & 2 & 170 \\
& $M 1$ & 465.4 & 100 & $6.282 \times 10^{-2}$ & $5.42 \times 10^{-9}$ & 4 & 117 \\
\hline $19 / 2^{+}$ & $E 1$ & $(22.7)^{a}$ & $(884.1)^{b}$ & $0.3932 \times 10^{1}$ & $3.92 \times 10^{-6}$ & 2 & $505 /(5.1)^{c}$ \\
& $E 1$ & 465.4 & 100 & $7.991 \times 10^{-3}$ & $5.17 \times 10^{-11}$ & 4 & $373 /(37)^{c}$ \\
\hline $21 / 2^{+}$ & $M 2$ & $(22.7)^{a}$ & $(0.494)^{b}$ & $8.834 \times 10^{3}$ & $1.94 \times 10^{1}$ & 2 & 0.23 \\
& $M 2$ & 465.4 & 100 & $2.004 \times 10^{-1}$ & $1.09 \times 10^{-3}$ & 4 & 5.5 \\
\hline $21 / 2^{-}$ & $E 2$ & $(22.7)^{a}$ & $(1.16)^{b}$ & $3.758 \times 10^{3}$ & $4.35 \times 10^{-1}$ & 2 & 1.5 \\
& $E 2$ & 465.4 & 100 & $2.417 \times 10^{-2}$ & $1.04 \times 10^{-5}$ & 4 & 18 \\
\hline \hline
\end{tabular}

\footnotetext{
${ }^{a}$ Energy of unobserved transition.

${ }^{b}$ Intensity of unobserved transition inferred from intensity balance and implied conversion coefficients.

${ }^{c}$ Entries in italics include a normalisation factor of $10^{4}$ (see text for details).
}

treme values of the reduced hindrances can be used to rule out the assumed spin and parity.

At first glance, reasonable values for the reduced hindrances (expected range 30-300) are only obtained for a $19 / 2^{-}$assignment to the isomeric state. However, for $\mathrm{J}^{\pi}=19 / 2^{-}$there are potential E2 transitions of 703 and $230 \mathrm{keV}$ that should directly depopulate the isomer and feed the 629 and $1102 \mathrm{keV}$ states, respectively. Such transitions were not observed in any of the (various) out-ofbeam, double-gated spectra or in the delayed spectrum in Figure 1(c). Hence, upper intensity limits were deduced, giving limits on the reduced hindrances for the possible 703 and $230 \mathrm{keV}$ transitions of $f_{v}>103$ and $f_{v}>6900$ respectively. The latter limit effectively rules out the $19 / 2^{-}$ assignment. The $21 / 2^{+}$possibility can be ruled out on the basis of the very low values of the reduced hindrances for both the 465 and $23 \mathrm{keV}$ transitions that fall outside the expected range mentioned above.

For the 21/2- alternative, both notional E2 decays of 23 and $465 \mathrm{keV}$ are fast, however, they could have been enhanced by mixing between the isomer and the $21 / 2^{-}$state in the $9 / 2^{-}$band, resulting in the low reduced hindrances. If the entire strength of the $465 \mathrm{keV}$ transition were due to a collective admixture of the $K^{\pi}=9 / 2^{-}$wavefunction into the isomeric state wavefunction, a mixing matrix element of $23 \mathrm{eV}$ would be implied, as deduced using the method described in [7], a reasonable value. However, in this scenario, there should also be a $203 \mathrm{keV} \mathrm{M1/E2} \mathrm{tran-}$ sition from the isomer to the known $19 / 2^{-}$band member at $1131 \mathrm{keV}$ [13], with a branching ratio relative to the $465 \mathrm{keV}$ transition that is consistent with the collective properties expected (and observed) in the 9/2- [514] band.

Table 2 presents the corresponding magnetic moment properties deduced from the measured crossover/cascade intensity ratios within this band. The values deduced for $\left|g_{K}-g_{R}\right|$ are consistent with the previous measurement [13], theoretical expectations $\left(g_{K}=1.29\right.$ for the $9 / 2^{-}[514]$ configuration and $\left.\mathrm{g}_{R} \sim 0.35\right)$ and with branching ratios in the same band in the neighbouring nucleus, ${ }^{185} \mathrm{Ta}[11$, 12]. For the decays from the isomer to reproduce the average $\left|g_{K}-g_{R}\right|$ for the inband decays, the intensity ratio $\lambda=I_{\gamma}(\Delta I=2 ; 465) / I_{\gamma}(\Delta I=1 ; 203)$ would have to be $1.07(13)$. The observed limit is $\lambda>2.57$. While this tends to discount the $21 / 2^{-}$possibility, it still cannot be ruled out.

The remaining possibility for the spin assignment is $19 / 2^{+}$. Given the known variability in $E 1$ strengths, the measured reduced hindrances for the 23 and the $465 \mathrm{keV}$ transitions are within acceptable limits, as are the limits for the possible 703 and $230 \mathrm{keV} \mathrm{M2}$ transitions of $f_{v}>22$ and $f_{v}>67$, respectively. Based on the above discussions, it can be concluded that $19 / 2^{+}$or $21 / 2^{-}$are the likely assignments for the $1.3 \mu \mathrm{s}$ isomer.

\subsection{Configuration assignment}

Given the excitation energy of the isomer, a 3-quasiparticle configuration is probable. In the light odd mass tantalum isotopes, 21/2- isomers are observed systematically across the isotopic chain. Their nature is discussed in detail by Kondev et al [17] for isotopes with $\mathrm{A} \leq 179$, while the isomer persists into ${ }^{181} \mathrm{Ta}[18,19]$. Although mixing of specific configurations is likely, particularly for the $A=179$ case [17], a three-proton configuration is a possible candidate for the isomer configuration in ${ }^{183} \mathrm{Ta}$. Note that in ${ }^{185} \mathrm{Ta}$, the $17 \mathrm{~ms}, 21 / 2^{-}$isomer has been assigned as the $\pi 7 / 2^{+}[404] \otimes v 3 / 2^{-}[512] 11 / 2^{+}[615]$ configuration in [11], however, the three proton configuration has also been suggested as an alternative [21] based on the transition strength systematics. In ${ }^{183} \mathrm{Ta}$, all possibilities must be considered, including the fact that $19 / 2^{+}$appears to be the more likely spin and parity assignment.

The neutron states most likely to be involved in the configuration can be determined by examining the lowlying spectrum of excited states in the nearby odd-mass tungsten isotopes, especially ${ }^{183} \mathrm{~W}[20]$ and ${ }^{185} \mathrm{~W}$ [22]. The ground state in ${ }^{183} \mathrm{~W}$ is formed from the $1 / 2^{-}$[510] intrisic state, with the $3 / 2^{-}$[512] state only $209 \mathrm{keV}$ higher.

Table 2. Measured in-band branching ratios for the $9 / 2^{-}[514]$ band in ${ }^{183} \mathrm{Ta}$, together with inferred gyromagnetic ratios.

\begin{tabular}{|c|c|c|c|c|}
\hline$J_{i}^{\pi}$ & $\begin{array}{c}E_{\gamma} \\
(I \rightarrow I-1) \\
(\mathrm{keV})\end{array}$ & $\begin{array}{c}E_{\gamma} \\
(I \rightarrow I-2) \\
(\mathrm{keV})\end{array}$ & $\lambda^{a}$ & $\left|g_{K}-g_{R}\right|^{b}$ \\
\hline $13 / 2^{-}$ & 186 & 343 & $0.14(3)$ & $0.83(9)$ \\
\hline $15 / 2^{-}$ & 212 & 397 & $0.25(4)$ & $0.93(7)$ \\
\hline \multirow[t]{2}{*}{$17 / 2^{-}$} & 238 & 450 & $0.45(6)$ & $0.89(6)$ \\
\hline & & weig & ted avg: & $0.89(4)$ \\
\hline
\end{tabular}

${ }^{a} \lambda=I_{\gamma}(I \rightarrow I-2) / I_{\gamma}(I \rightarrow I-1)$.

${ }^{b}$ Calculations assume a value of $Q_{0}=6.5 \mathrm{eb}$. 
Table 3. Expected low-lying 3-quasiparticle configurations.

\begin{tabular}{ccccc}
\hline \hline$K^{\pi}$ & Configurations $^{a}$ & $\begin{array}{c}\mathrm{E}_{q p} \\
(\mathrm{keV})\end{array}$ & $\begin{array}{c}\mathrm{E}_{\text {res }} \\
(\mathrm{keV})\end{array}$ & $\begin{array}{c}\mathrm{E}_{\text {cal }} \\
(\mathrm{keV})\end{array}$ \\
\hline $19 / 2^{-}$ & $v 1 / 2^{-} 11 / 2^{+} \pi 7 / 2^{+}$ & 1930 & +263 & 2193 \\
$17 / 2^{-}$ & $v\left(1 / 2^{-}\right) 11 / 2^{+} \pi 7 / 2^{+}$ & & -137 & 1793 \\
\hline $21 / 2^{+}$ & $v 1 / 2^{-} 11 / 2^{+} \pi 9 / 2^{-}$ & 2097 & -64 & 2033 \\
$19 / 2^{+}$ & $v\left(1 / 2^{-}\right) 11 / 2^{+} \pi 9 / 2^{-}$ & & -222 & 1875 \\
\hline $21 / 2^{-}$ & $v 3 / 2^{-} 11 / 2^{+} \pi 7 / 2^{+}$ & 1984 & -138 & 1846 \\
\hline $23 / 2^{+}$ & $v 3 / 2^{-} 11 / 2^{+} \pi 9 / 2^{-}$ & 2151 & -216 & 1935 \\
\hline $21 / 2^{-}$ & $\pi 5 / 2^{+} 7 / 2^{+} 9 / 2^{-}$ & 1406 & -77 & 1329 \\
\hline \hline
\end{tabular}

a Neutrons: $1 / 2^{-}: \quad 1 / 2^{-}[510] ; \quad 3 / 2^{-}: \quad 3 / 2^{-}[512]$; $11 / 2^{+}: 11 / 2^{+}[615]$. Protons: $5 / 2^{+}: 5 / 2^{+}[402] ; 7 / 2^{+}: 7 / 2^{+}[404]$; $9 / 2^{-}: 9 / 2^{-}[514]$. An orbital in brackets, e.g. $\left(1 / 2^{-}\right)$, refers to an anti-parallel coupling.

This situation is reversed in ${ }^{185} \mathrm{~W}$, with the $1 / 2^{-}[510]$ level at an excitation energy of only $24 \mathrm{keV}$, while the $11 / 2^{+}[615]$ state lies at 310 and $197 \mathrm{keV}$ in ${ }^{183} \mathrm{~W}$ and ${ }^{185} \mathrm{~W}$, respectively. The $7 / 2^{-}[503]$ and $9 / 2^{+}[624]$ levels are higher in energy and therefore are not expected to take part in the formation of low-lying two-neutron oneproton states in odd-mass tantalum nuclei. As discussed above, the $\pi 5 / 2^{+}[402] 7 / 2^{+}[404] 9 / 2^{-}[514]$ configuration will also be competitive in energy.

To quantify the situation, preliminary multiquasiparticle calculations were performed for ${ }^{183} \mathrm{Ta}$, as well as for ${ }^{181} \mathrm{Ta}$ and ${ }^{185} \mathrm{Ta}$ to investigate the isotopic dependence. The results are illustrated in figure 3 , and the detailed energies for ${ }^{183} \mathrm{Ta}$ are given in table 3 . The calculated energy dependence for the $\pi 5 / 2^{+}[402] 7 / 2^{+}[404] 9 / 2^{-}[514], 21 / 2^{-}$state is flat (labelled $\mathrm{F}$ in figure 3 ). The $21 / 2^{-}$state from the $v 3 / 2^{-}[512] 11 / 2^{+}[615] \otimes \pi 7 / 2^{+}[404]$ configuration (labelled G) comes down in energy with increasing mass number, becoming more competitive in ${ }^{185} \mathrm{Ta}$. If the state in ${ }^{183} \mathrm{Ta}$ were to be $21 / 2^{-}$, it would have the $\pi 5 / 2^{+}[402] 7 / 2^{+}[404] 9 / 2^{-}[514]$ configuration. However, the energy of the the $19 / 2^{+}$state in ${ }^{183} \mathrm{Ta}(1875 \mathrm{keV}$ in table 3 ) is clearly over-estimated in the calculation given that the $5^{-}$two neutron component of the configuration is observed in the $\mathrm{N}=110$ isotone, ${ }^{184} \mathrm{~W}$, at $1285 \mathrm{keV}$ [23]. If the state in ${ }^{183} \mathrm{Ta}$ were to be $19 / 2^{+}$, this would imply that $v 1 / 2^{-}[510] 11 / 2^{+}[615] \otimes \pi 9 / 2^{-}[514]$ is the likely configuration.

To distinguish between these possibilities requires either better spectroscopic information for the $465 \mathrm{keV}$ direct decay, or observation of the characteristic band structure above the isomer. However, preliminary analysis of the states above the isomer does not result in a well developed band structure. This is perhaps not unexpected given the high density of intrinsic states predicted to occur in the vicinity of the isomer, as shown in figure 3 .

\section{Conclusion}

Excited states in neutron-rich tantalum isotopes have been studied using deep-inelastic reactions between a ${ }^{136} \mathrm{Xe}$ beam and a ${ }^{186} \mathrm{~W}$ target. The energy of the isomeric state in ${ }^{183} \mathrm{Ta}$ previously reported by Shizuma et al [13] has now been established on the basis of a $\gamma$-ray transition

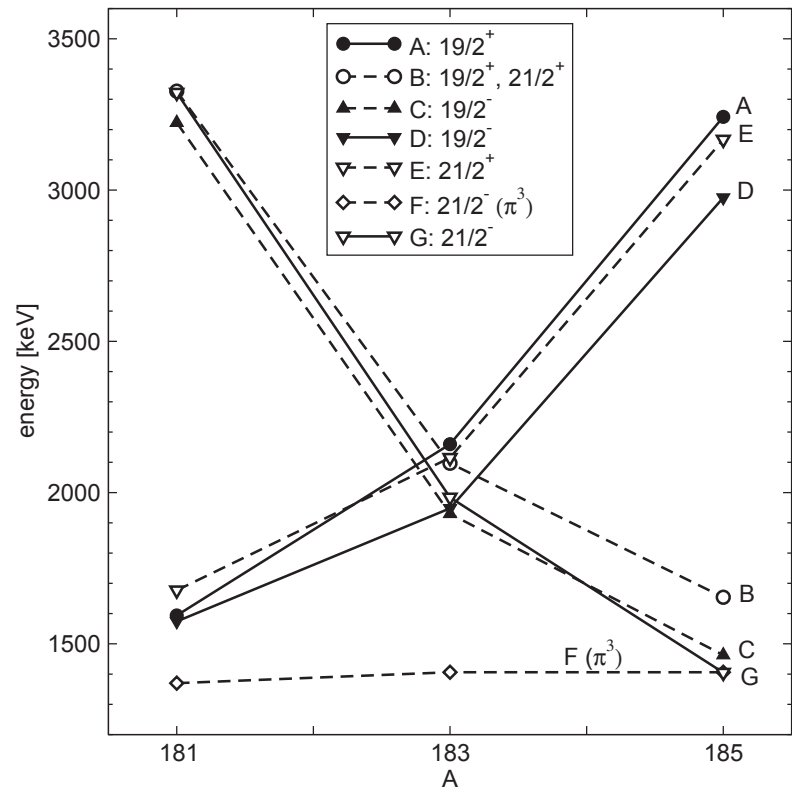

Fig. 3. Systematic behaviour between $A=181$ and $A=185$ for the lowest intrinsic states calculated in ${ }^{183} \mathrm{Ta}$.(preliminary calculations)

that directly depopulates it. The measured reduced hindrances for the depopulating transitions support either a $19 / 2^{+}$or $21 / 2^{-}$assignment. The $19 / 2^{+}$possibility is currently favoured, in which case a 3 -quasiparticle $\pi 9 / 2^{-}$[514] $\otimes v 1 / 2^{-}[510] 11 / 2^{+}[615]$ configuration is suggested.

\section{References}

1. P. M Walker and G.D. Dracoulis, Nature 399 (1999) 35.

2. P. M Walker and G.D. Dracoulis, Hyperfine Interactions 135 (2001) 83.

3. R. D'Alarcao et al, Phys. Rev. C 59 (1999) R1227.

4. G. J. Lane et al, Phys. Rev. C 82 (2010) 052304(R).

5. G. F. Farrelly et al., Acta Phys. Pol. B 40 (2009) 885.

6. G. D. Dracoulis et al, Phys. Lett. B 584 (2004) 22.

7. G. D. Dracoulis et al, Phys. Rev. Lett. 97 (2006) 122501 .

8. C. Wheldon et al J. Phys. G: Nucl. Part. Phys. 27 (2001) L13.

9. R. Broda, J. Phys. G: Nucl. Part. Phys. 32 (2006) R151.

10. M. Pfutzner et al Phys. Lett. B 444, (1998) 32.

11. G. J. Lane et al, Phys. Rev. C 80, (2009) 024321.

12. C. Wheldon et al, Eur. Phys. J. A 5, (1999) 353-355.

13. T. Shizuma et. al, Eur. Phy. J. A 39, (2009) 263.

14. G. D. Dracoulis et al, Phys. Lett. B 709 (2012) 59.

15. F. G. Kondev et al, Phys. Rev. C 80 (2009) 014304

16. M. Cromaz et al, Nucl. Instrum. Methods A 462, (2001) 519.

17. F. G. Kondev et al, Nucl. Phys. A 617 (1997) 91.

18. C. Wheldon et al, Phys. Lett. B 425 (1998) 239.

19. G. D. Dracoulis et al Phys. Rev. C 58 (1998) 1837.

20. T. R. Saitoh et al, Nucl Phys A 669 (2000) 381.

21. P. M Walker et al, Phys. Rev. C 81 (2010) 041304(R)

22. V. Bondarenko et al, Nucl. Phys. A 762 (2005) 167.

23. C. Wheldon et al, Eur Phys. J. A 20 (2004) 365. 